\title{
MeHg-induced autophagy via JNK/Nps34 complex pathway promotes autophagosome accumulation and neuronal cell death
}

\author{
Tianji Lin ${ }^{1}$, Shijuan Ruan ${ }^{1}$, Dingbang Huang ${ }^{1}$, Xiaojing Meng ${ }^{1}$, Wenjun $\mathrm{Li}^{1}$, Bin Wang ${ }^{1}$ and Fei Zou ${ }^{1}$
}

\begin{abstract}
Methylmercury (MeHg), an environmental toxin, may specifically cause neurological disorders. Recent studies have reported that autophagy can be induced by metals and be involved in metal cytotoxicity. However, the role of autophagy in MeHg-induced neurotoxicity remains unknown. Here, we demonstrate that MeHg induces mTORindependent autophagy through JNKVps34 complex pathway, which further promotes autophagosome accumulation and neuronal cell death. In addition to cell death, MeHg increased LC3-II expression in a concentrationand time-dependent manner in neuronal cells; furthermore, western blot analysis of LC3-II expression under baf A1treated condition indicates that MeHg activates autophagy induction. However, we found lysosomal degradative function was impaired by MeHg. Under this condition, MeHg-activated autophagy induction would elicit autophagosome accumulation and cell death. Consistent with this inference, the autophagy inhibitor decreased the MeHg-induced autophagosome accumulation and neuronal cells death, whereas the autophagy inducers further augmented MeHg cytotoxicity. Then, the mechanism of autophagy induction is investigated. We show that MeHginduced autophagy is mTOR-independent. Vacuolar protein sorting 34 (Vps34) complex is critical for mTORindependent autophagy. MeHg induced the interaction between Beclin1 and Vps34 to form Vps34 complex. Importantly, knockdown of Vps34 inhibited autophagy induction by MeHg. Furthermore, we found that JNK, but not p38 or ERK, promoted the formation of Vps34 complex and autophagy induction. Finally, inhibition of JNK or downregulation of Vps34 decreased autophagosome accumulation and alleviated MeHg-induced neuronal cell death. The present study implies that inhibiting JNKNps34 complex autophagy induction pathway may be a novel therapeutic approach for the treatment of MeHg-induced neurotoxicity.
\end{abstract}

\section{Introduction}

Mercury has been continually discharged from natural sources as well as industrial activities in recent years ${ }^{1}$. Microorganisms are able to transform inorganic mercury to $\mathrm{MeHg}$, then $\mathrm{MeHg}$ accumulates in plants and animals.

\footnotetext{
Correspondence: Bin Wang (wenwunj@smu.edu.cn) or

Fei Zou (zfei@smu.edu.cn)

${ }^{1}$ Department of Occupational Health and Occupational Medicine, School of Public Health, Southern Medical University, 510515 Guangzhou, Guangdong, China

These authors contributed equally: Tianji Lin, Shijuan Ruan

Edited by B. Zhivotovsky
}

In this way, MeHg reaches the food chain, which is dangerous for humans ${ }^{2}$. MeHg can cross the blood-brain barrier to accumulate in the central nervous system $(\mathrm{CNS})^{3}$. MeHg induces neurotoxic effects that lead to developmental deficits such as loss of intelligence quotient (IQ) points, reduction in language skills, impaired memory function, and attention deficits in children exposed in-utero ${ }^{4}$.

Several studies have reported that autophagy can be induced by starvation, nutrient depletion, and environmental stress ${ }^{5,6}$. Under physiological condition, autophagy

\section{(c) The Author(s) 2019}

(c) (i) Open Access This article is licensed under a Creative Commons Attribution 4.0 International License, which permits use, sharing, adaptation, distribution and reproduction c. in any medium or format, as long as you give appropriate credit to the original author(s) and the source, provide a link to the Creative Commons license, and indicate if changes were made. The images or other third party material in this article are included in the article's Creative Commons license, unless indicated otherwise in a credit line to the material. If material is not included in the article's Creative Commons license and your intended use is not permitted by statutory regulation or exceeds the permitted use, you will need to obtain permission directly from the copyright holder. To view a copy of this license, visit http://creativecommons.org/licenses/by/4.0/. 
protects cell from death through sequestering damaged protein and organelles into autophagosomes formed by autophagy induction and delivering autophagosomes to lysosomes for degradation. In the studies on the neurotoxicity of certain metal (such as cadmium and manganese), autophagy can protect neuronal cell from death ${ }^{7,8}$. However, autophagy induction can also serve a pro-death role in pathological conditions. In vivo and in vitro, studies have reported that autophagy induction promotes cytotoxicity through excessive elimination of intracellular organelles and cytosol ${ }^{9,10}$ or autophagosome accumulation $^{11-14}$. What role autophagy plays in MeHg-induced neurotoxicity remains unclear.

Autophagy is induced by mTOR-dependent or mTORindependent pathway ${ }^{15}$. The mTOR-dependent autophagy is negatively regulated by $\mathrm{mTOR}^{16}$. In addition to nutrient starvation, the neurotoxin has been found to induce autophagy via mTOR-dependent pathway ${ }^{17}$. In mTOR-independent autophagy, Vps34 complex is a critical mediator for autophagy induction ${ }^{18}$. Beclin1 binds to Vps34 to form the core Vps34 complex to activate autophagy induction. It is not known whether the MeHginduced autophagy is mTOR-dependent or mTORindependent.

MAPKs (mitogen-activated protein kinases) have been reported to be related to autophagy induction and play an important role in neurotoxicity ${ }^{19-21}$. The mammalian MAPK family consists of ERK (extracellular signalregulated kinase), p38, and JNK (c-Jun NH2-terminal kinase). Various observations suggest that MAPKs contribute to metals-induced neurotoxicity ${ }^{22,23}$. However, how MAPKs regulate autophagy induction and cell death in $\mathrm{MeHg}$-induced neurotoxicity are not understood.

In this study, we demonstrate that $\mathrm{MeHg}$ induces mTOR-independent autophagy through JNK/Vps34 complex pathway, which further promotes autophagosome accumulation and neuronal cell death.

\section{Results}

\section{MeHg induces neuronal cell death}

We used SH-SY5Y cells and rat cerebral cortical neurons to study $\mathrm{MeHg}$-induced neuronal cell death. As shown in Fig. 1a, treatment with $\mathrm{MeHg}$ for $24 \mathrm{~h}$ caused a concentration-dependent increase of apoptosis in $\mathrm{SH}$ SY5Y cells. Furthermore, MeHg increased cleaved fragments of caspase-9 and PARP (apoptosis markers) at $24 \mathrm{~h}$ (Fig. 1b). Moreover, in rat cerebral cortical neurons, $\mathrm{MeHg}$ induced cell death and impaired synapse in a dosedependent manner at $12 \mathrm{~h}$ (Fig. 1c). These results indicate that $\mathrm{MeHg}$ induces neuronal cell death.

\section{MeHg increases autophagy induction in neuronal cells}

Autophagy is known as one of the critical cellular homeostatic mechanisms and closely related to cell death.
LC3-II is the most commonly used marker of autophagy induction $^{24}$. As shown in Fig. 2a, b, MeHg induced LC3-II protein expression in the concentration- and timedependent manner in SH-SY5Y cells. To further determine whether $\mathrm{MeHg}$ activated autophagy induction, bafilomycin A1 (baf A1) was used to inhibit autophagic degradation for $4 \mathrm{~h}$ before sample collection. We found that $\mathrm{MeHg}$ induced LC3-II expression more significantly compared with control group during $0-4 \mathrm{~h}$ and $20-24 \mathrm{~h}$ under baf A1-treated condition (Fig. 2c). Similar results were observed in baf A1-treated rat cerebral cortical neurons during $8-12 \mathrm{~h}$ after $\mathrm{MeHg}$ exposure (Fig. 2d). These findings suggest that $\mathrm{MeHg}$ increases autophagy induction in neuronal cells.

\section{Autophagy induction promotes autophagosome accumulation and cell death in MeHg-exposed neuronal cells}

Once stress activates autophagy induction to form autophagosome, the following efficient degradation of autophagy substrates (protein aggregates and damaged organelles) is critical for maintaining cellular homeostasis $^{25}$. To ascertain the role of autophagy induction in MeHg-induced cell death, lysosomal degradative function was therefore determined through western blot analysis of p62 protein expression. An increase in p62 was observed in cerebral cortical neurons treated with $2 \mu \mathrm{M} \mathrm{MeHg}$ for $12 \mathrm{~h}$ (Fig. 3a). Moreover, the autophagic inducers Torin 1 $(1 \mu \mathrm{M})$ and trehalose $(5 \mathrm{mM})$ decreased p62 protein expression more markedly in control group compared with that in MeHg-treated SH-SY5Y cells (Fig. 3b). These results indicate that $\mathrm{MeHg}$ impairs the autophagic degradation, and under this condition, increased autophagy induction would elicit autophagosome accumulation.

To further investigate the effect of autophagy induction on autophagosome accumulation, SH-SY5Y cells were pretreated with autophagy inhibitor or inducer, respectively. The AAV-mRFP-GFP-LC3 introduced into neuronal cells was used to detect autophagosome accumulation $^{26,27}$. MeHg increased the number and the size of the yellow puncta (autophagosomes) in SH-SY5Y cells at $4 \mathrm{~h}$ and $24 \mathrm{~h}$, which indicates that $\mathrm{MeHg}$ induces autophagosome accumulation (Fig. 3c). Importantly, wortmannin (autophagy inhibitor) decreased the number of autophagosomes in MeHg-treated SH-SY5Y cells, whereas Torin 1 or trehalose (autophagy inducer) further increased the number and size of the autophagosomes (Fig. 3d). These results suggest that $\mathrm{MeHg}$-induced autophagy promotes autophagosome accumulation.

Finally, neuronal cells were pretreated with autophagic inhibitor or inducer to further ascertain the role of $\mathrm{MeHg}$ induced autophagy in neuronal cell death. Wortmannin decreased the apoptosis in MeHg-treated SH-SY5Y cells, 


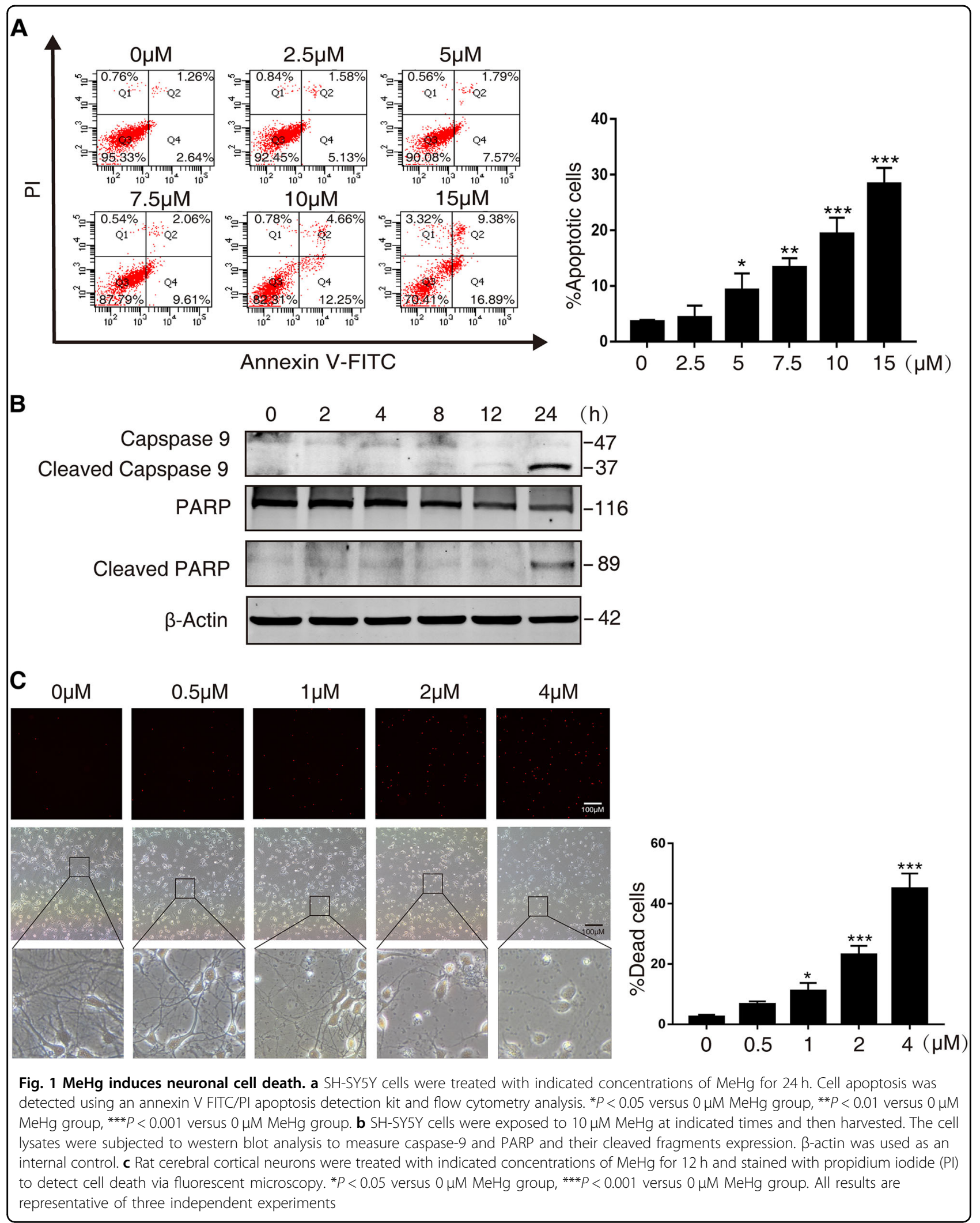



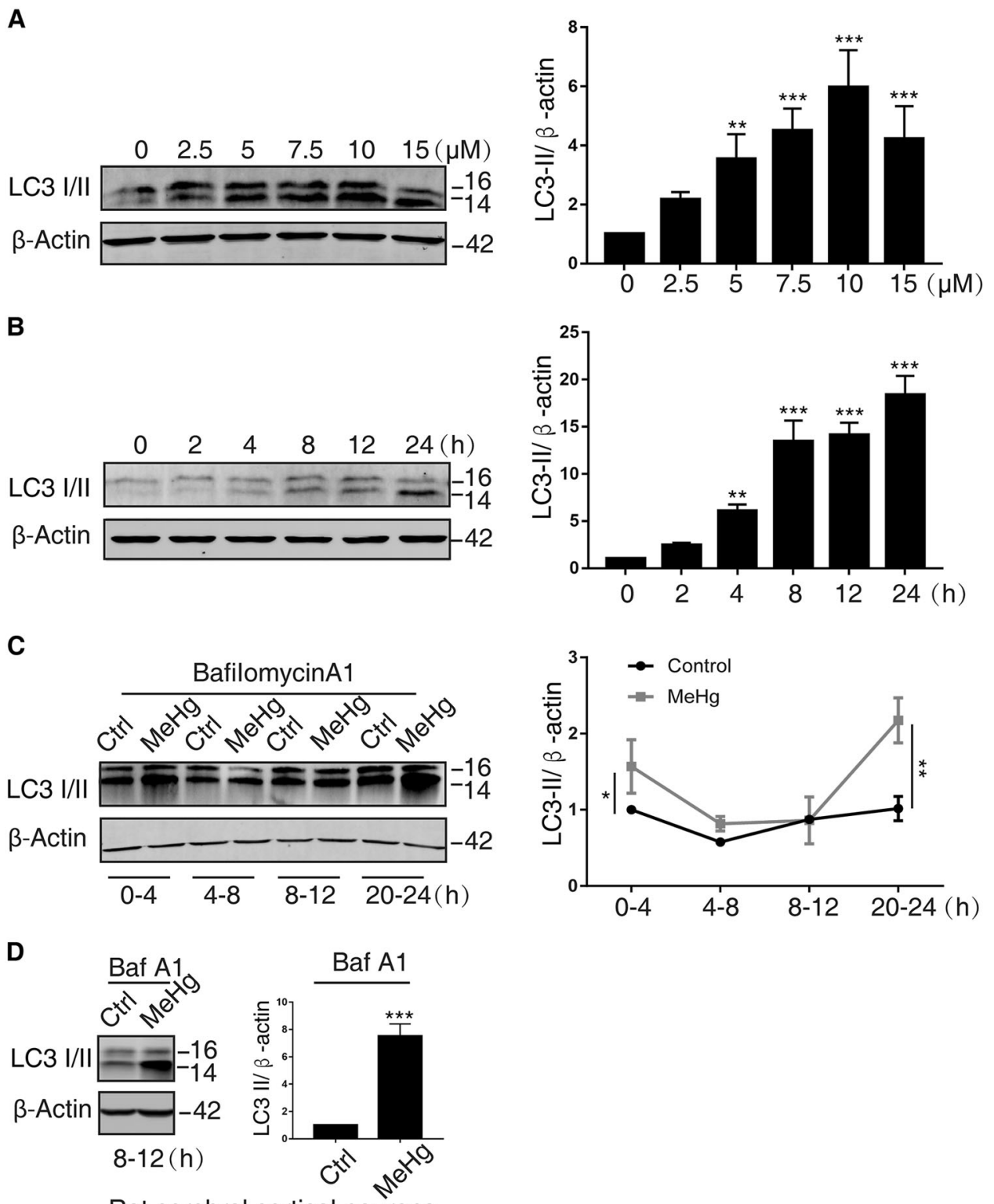

Rat cerebral cortical neurons

Fig. 2 MeHg induces LC3 protein expression in both SH-SY5Y cells and rat cerebral cortical neurons. a SH-SY5Y cells were treated with indicated concentrations of MeHg for $24 \mathrm{~h}$ and then harvested. The cell lysates were subjected to western blot analysis to measure LC3-II expression. ${ }^{* *} P<0.01$ versus $0 \mu \mathrm{M}$ MeHg group, ${ }^{* *} P<0.001$ versus $0 \mu \mathrm{M}$ MeHg group. b SH-SY5Y cells were exposed to $10 \mu \mathrm{M}$ MeHg at indicated times and then harvested. The cell lysates were subjected to western blot analysis to measure LC3-II expression. ${ }^{* *} P<0.01$ versus $0 \mu \mathrm{M}$ MeHg group, ${ }^{* * *} P<$ 0.001 versus $0 \mu \mathrm{M}$ MeHg group. c MeHg-exposed $(10 \mu \mathrm{M}$ ) SH-SY5Y cells were treated with bafilomycin A1 (baf A1; $400 \mathrm{nM}$ ) for $4 \mathrm{~h}$ prior to sample processing at different time point. The cell lysates were subjected to western blot analysis to measure LC3-II expression. ${ }^{*} P<0.05$ versus $0-4 \mathrm{~h}$ control group, ${ }^{*} P<0.01$ versus $20-24 \mathrm{~h}$ control group. $\mathbf{d}$ MeHg-exposed $(2 \mu \mathrm{M})$ rat cerebral cortical neurons were treated with bafilomycin A1 (baf A1; $400 \mathrm{nM}$ ) for $4 \mathrm{~h}$ prior to sample processing at $12 \mathrm{~h}$. The cell lysates were subjected to western blot analysis to measure LC3-II expression. ${ }^{* * *} P<0.001$ versus $8-12 \mathrm{~h}$ control group. All results are representative of three independent experiments

whereas Torin 1 and trehalose augmented MeHg-induced apoptosis (Fig. 3e). Furthermore, the cleaved fragments of caspase- 9 and PARP were decreased by wortmannin and increased by Torin 1 or trehalose (Fig. 3f). In rat cerebral cortical neurons, the effects of autophagic inhibitor or inducer on $\mathrm{MeHg}$-induced cytotoxicity were similar to those observed in the SH-SY5Y cells (Fig. 3g). These findings indicate that $\mathrm{MeHg}$-activated autophagy induction promotes autophagosome accumulation and neuronal cell death. 


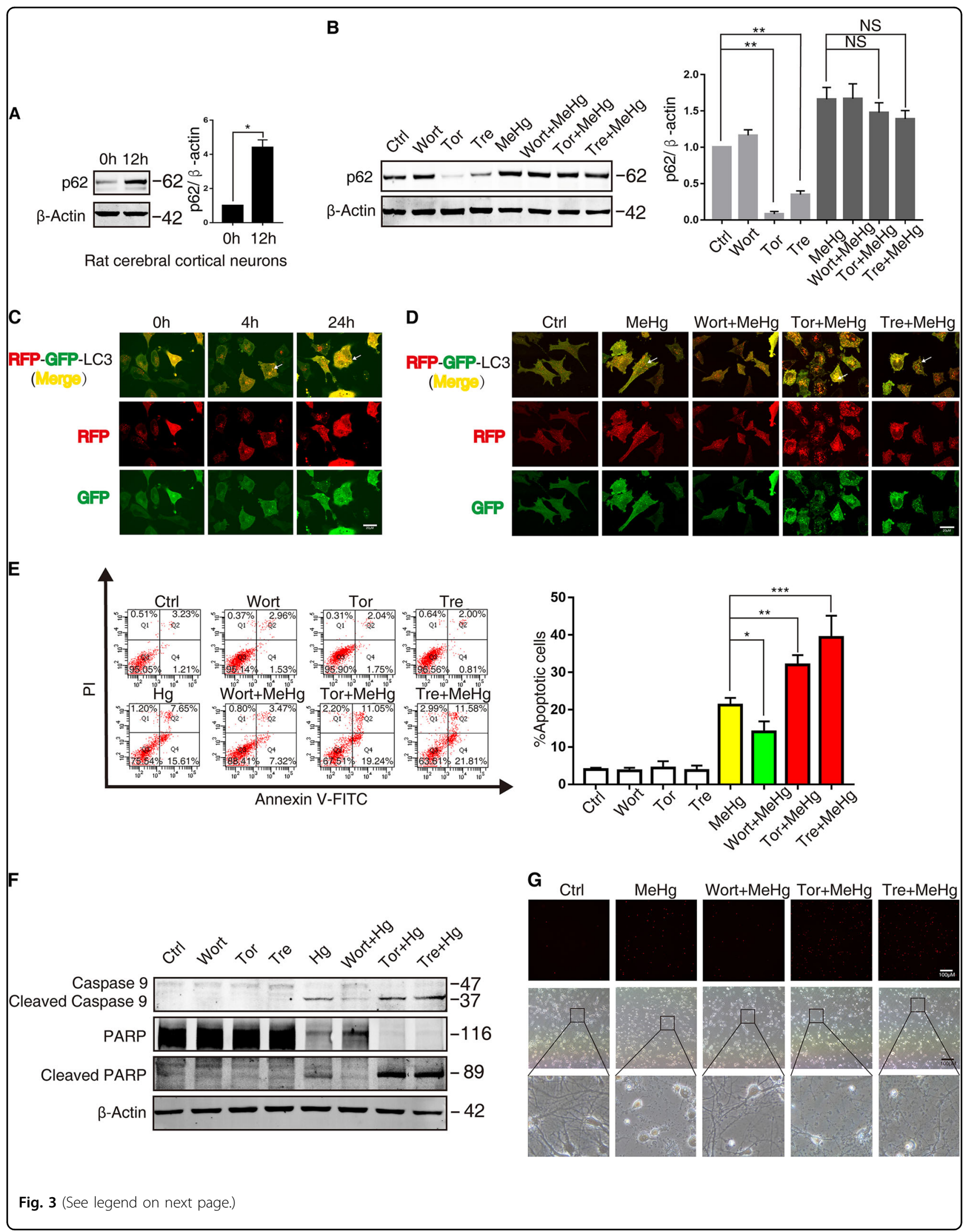




\begin{abstract}
(see figure on previous page)
Fig. $3 \mathrm{MeHg}$ leads to autophagosome accumulation and neuronal cell death. a Rat cortical neurons were treated with $2 \mu \mathrm{M}$ MeHg for $12 \mathrm{~h}$. The cell lysates were subjected to western blot analysis to measure p62 expression. ${ }^{*} P<0.05$ versus $0 \mathrm{~h}$ group. b SH-SY5Y cells were pretreated with wortmannin (wort; $1 \mu \mathrm{M} 1 \mathrm{~h}$ ), Torin 1 (Tor; $1 \mu \mathrm{M} 1 \mathrm{~h}$ ), or trehalose (tre; $50 \mathrm{mM} 1 \mathrm{~h}$ ), then the cells were exposed to $10 \mu \mathrm{M}$ MeHg for $24 \mathrm{~h}$. The cell lysates were subjected to western blot analysis to measure p62 expression. ${ }^{*} P<0.01$ versus control group, NSå 0.05 versus MeHg group. c SH-SY5Y cells were infected with adeno-associated viral vector (AAV-mRFP-GFP-LC3) at final MOI 100 for $24 \mathrm{~h}$. Then cells were exposed to $10 \mu \mathrm{M} \mathrm{MeHg}$ at indicated times. The cells were examined by confocal microscopy $(\times 100)$. $\mathbf{d}$ After AAV-mRFP-GFP-LC3 infection, the protocol was followed as described in $\mathbf{b}$. Then the SH-SY5Y cells were examined by confocal microscopy $(\times 100)$. e The protocol was followed as described in $\mathbf{b}$. Cell apoptosis was detected using an annexin V FITC/PI apoptosis detection kit and flow cytometry analysis. ${ }^{*} P<0.05$ versus MeHg group, ${ }^{* *} P<0.01$ versus MeHg group, ${ }^{* * *} P<$ 0.001 versus MeHg group. f The protocol was followed as described in $\mathbf{b}$. The cell lysates were subjected to western blot analysis to measure caspase9 and PARP and their cleaved fragments expression. $\mathbf{g}$ Rat cerebral cortical neurons were pretreated with wortmannin (wort; $100 \mathrm{nM} 1 \mathrm{~h}$ ), Torin1 (Tor; $250 \mathrm{nM} 1 \mathrm{~h}$ ), or trehalose (tre; $50 \mathrm{mM} 1 \mathrm{~h}$ ), then the cells were exposed to $2 \mu \mathrm{M} \mathrm{MeHg}$ for $12 \mathrm{~h}$ and stained with propidium iodide (PI) to detect cell death via fluorescent microscopy. All results are representative of three independent experiments
\end{abstract}

\section{MeHg induces mTOR-independent autophagy through JNK/Vps34 complex signaling pathway}

Autophagy induction signaling involves mTORdependent and mTOR-independent pathways ${ }^{15}$. The mTOR-dependent autophagy is negatively regulated by mTOR. mTOR activity can be inferred by the levels of phosphorylation of its substrates (p70S6K and 4E-BP1) ${ }^{28}$. As shown in Fig. 4a, MeHg increased phosphorylation of p70S6K (Thr389, Ser371) and 4E-BP1 (Thr 37/46). Thus, $\mathrm{MeHg}$ induces mTOR-independent autophagy.

Vps34 has been reported to activate mTORindependent autophagy induction through binding Beclin1 to form the Vps34 core complex ${ }^{18}$. To determine whether $\mathrm{MeHg}$ induced interaction between Beclin1 and Vps34, Co-immunoprecipitation and immunofluorescence experiment were performed. As shown in Fig. 4b, MeHg enhanced the formation of Beclin1-Vps34 complex in SH-SY5Y cells. Consistently, MeHg increased Beclin1 (Fig. 4c, arrow a) and Vps34 (Fig. 4c, arrow b) colocalization (Fig. 4c, arrow c) detected by immunofluorescence in rat cerebral cortical neurons. To further address the role of Vps34 core complex in autophagy induction, chemically synthesized siRNAs against Vps34 were introduced into SH-SY5Y cells. Four siRNAs were screened and western blot analysis showed siRNA-1, siRNA-2, or siRNA-3 had little effect on downregulating Vps34 protein expression. However, Vps34 protein levels in SH-SY5Y cells transfected with siRNA-4 were reduced to $10 \%$ of that found in cultures transfected with the NC siRNA (Fig. 4d). Importantly, transfection with siRNA-4 reduced LC3-II induction in MeHg-exposed SH-SY5Y cells (Fig. 4e). These findings suggest that $\mathrm{MeHg}$ increases mTOR-independent autophagy induction through enhancing forming the Beclin1-Vps34 complex in neuronal cells.

MAPKs have been reported to be involved in mTORindependent autophagy ${ }^{29-31}$. Therefore, MAPKs activation was investigated following $\mathrm{MeHg}$ treatment. We found that MAPKs (ERK, p38, and JNK) were activated at different time by $\mathrm{MeHg}$ in SH-SY5Y cells (Fig. 4f). To further assess the effects of MAPKs on the VPS34 complex-dependent autophagy induction, SH-SY5Y cells were exposed to $\mathrm{MeHg}$ after pretreatment with MEK (upstream kinase of ERK) inhibitor U0126, p38 inhibitor SB203580, or JNK inhibitor SP600125 for $1 \mathrm{~h}$. Notably, pretreatment with SP600125 caused a dissociation of the Beclin1 and Vps34 complex (Fig. 4c, arrow e and f) and reduced LC3-II induction in MeHg-exposed SH-SY5Y cells (Fig. 4g). However, pretreatment with U0126 and SB203580 had little effect on VPS34 complex formation and LC3-II induction (Fig S1 a, b). Moreover, in rat cerebral cortical neurons, JNK was also activated with $2 \mu \mathrm{M}$ $\mathrm{MeHg}$ for $12 \mathrm{~h}$ (Fig. 4h). Immunofluorescence results showed that pretreatment with SP600125 disrupted the MeHg-induced colocalization between Beclin1 and Vps34. Thus, MeHg increases mTOR-independent autophagy induction through activating the JNK/Vps34 complex signaling pathway in neuronal cells.

\section{Downregulating JNK/Vps34 complex autophagy induction pathway can reduce the autophagosome accumulation and alleviates the neuronal cell death induced by $\mathrm{MeHg}$}

To further determine the effects of JNK/Vps34 complex pathway on autophagosome accumulation and neuronal cell death, neuronal cells were pretreated with SP600125 or transfected with siRNA. As shown in Fig. 5a, SP600125 decreased autophagosome accumulation (yellow puncta) in MeHg-exposed SH-SY5Y cells. Correspondingly, pretreatment with SP600125 suppressed the apoptosis and decreased the cleaved fragments of caspase 9 and PARP induced by MeHg in SH-SY5Y cells (Fig. 5b, c). However, pretreatment with U0126 and SB203580 had little effect on suppressing the MeHg-induced apoptosis of SH-SY5Y cells (Fig S2). Similar to results in SH-SY5Y cells, pretreatment with SP600125 suppressed cell death and synapse breakage induced by $\mathrm{MeHg}$ in rat cerebral cortical neurons (Fig. 5d). Moreover, transfection with siRNA against Vps34 also reduced MeHg-induced apoptosis and 


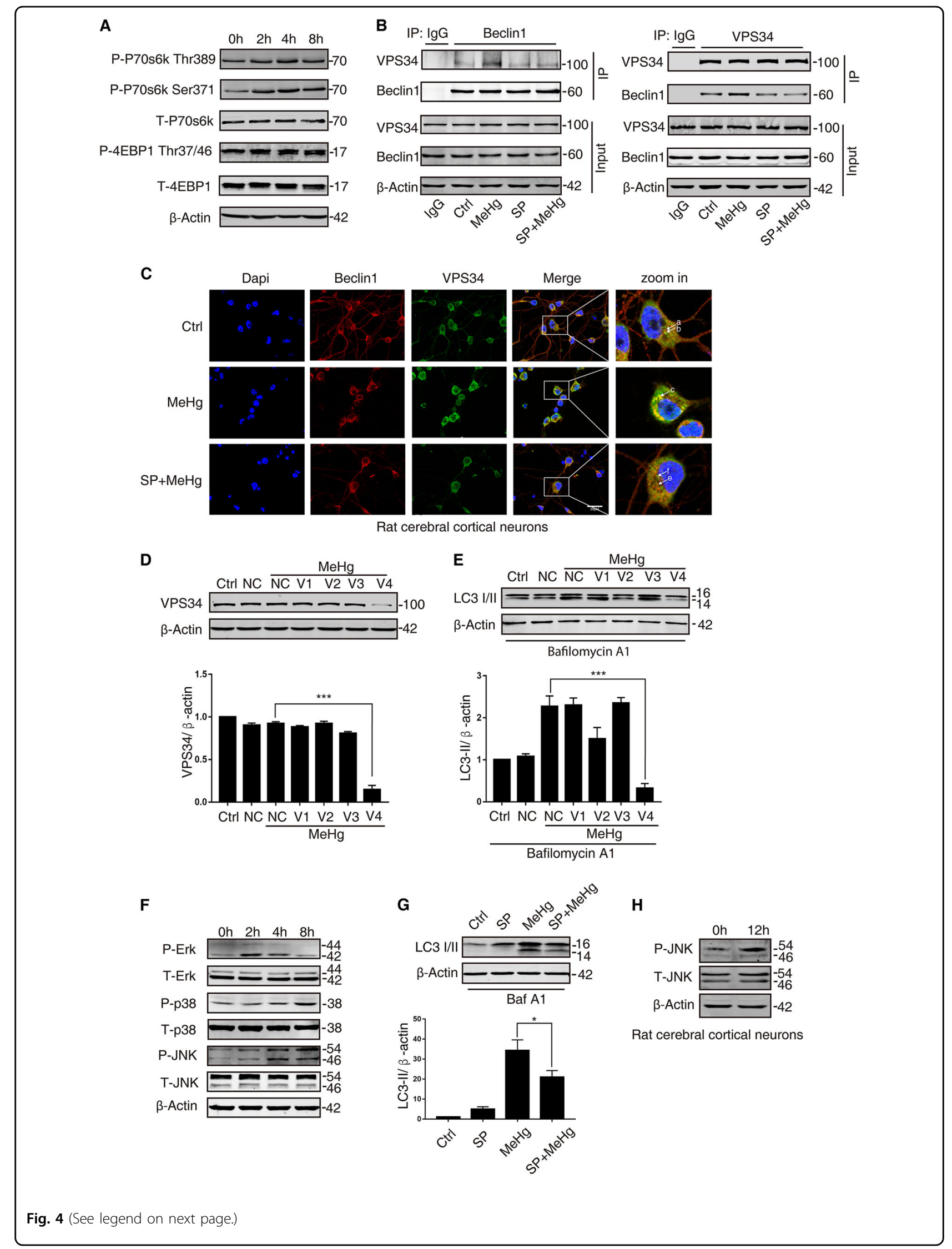


(see figure on previous page)

Fig. $4 \mathrm{MeHg}$ induces mTOR-independent autophagy through JNK/Vps34 complex signaling pathway. a SH-SY5Y cells were exposed to $10 \mu \mathrm{M}$ $\mathrm{MeHg}$ at indicated times. Phosphorylated and total mTOR substrate were detected by western blot. b SH-SY5Y cells were exposed to $10 \mu \mathrm{M}$ MeHg individually for $4 \mathrm{~h}$ or in combination with pretreatment of $30 \mu \mathrm{M} \mathrm{SP600125} \mathrm{for} 1 \mathrm{~h}$. The lysates were co-immunoprecipitated using a Beclin1 (Vps34)specific antibody, and the immunoprecipitants (pull down) were analysed by western blot for the presence of Vps34(Beclin1). The whole cell lysates (input) were analysed by western blot for Vps34 and Beclin1. c Rat cerebral cortical neurons were exposed to $2 \mu \mathrm{M} \mathrm{MeHg}$ individually for $12 \mathrm{~h}$ or in combination with pretreatment $2 \mu \mathrm{M}$ SP600125 for $1 \mathrm{~h}$. The cells were stained to determine the colocalization of Beclin 1 (red) and Vps34 (green). The cell nucleus was stained with DAPI (blue). $\mathbf{d}$ Negative control (NC) siRNA and Vps34 siRNAs (V1-V4) were transiently transfected into SH-SY5Y cells for $24 \mathrm{~h}$. Then, the cells were exposed to $10 \mu \mathrm{M}$ MeHg for $4 \mathrm{~h}$. Western blot analysis was performed to determine the Vps34 protein level. ${ }^{* * *} P<0.001$ versus MeHg-exposed negative control group. e The transfected cells exposed to MeHg were treated with bafilomycin A1 (baf A1, $400 \mathrm{nM}$ ) for $4 \mathrm{~h}$ before sample processing. LC3-II was detected by western blot. ${ }^{* *} P<0.001$ versus MeHg-exposed negative control group. f SH-SY5Y cells were exposed to $10 \mu \mathrm{M} \mathrm{MeHg}$ at indicated times and then harvested. Phosphorylated and total MAPK were detected by western blot. g SH-SY5Y cells were exposed to $10 \mu \mathrm{M}$ MeHg for $24 \mathrm{~h}$ after preincubation with JNK inhibitor $30 \mu \mathrm{M}$ SP600125 for $1 \mathrm{~h}$. Then, the cells were treated with bafilomycin A1 (baf A1, $400 \mathrm{nM}$ ) for $4 \mathrm{~h}$ before sample processing. LC3-II was detected by western blot. ${ }^{*} P<0.05$ versus MeHg group. $\mathbf{h}$ Rat cerebral cortical neurons were exposed to $10 \mu \mathrm{M}$ MeHg for $12 \mathrm{~h}$. Phosphorylated and total JNK were detected by western blot. All results are representative of three independent experiments

decreased the cleaved fragments of caspase 9 and PARP in MeHg-exposed SH-SY5Y cells. (Fig. 5e, f). Taken together, these findings suggest that downregulating JNK/ Vps34 complex autophagy induction pathway can reduce the autophagosome accumulation and alleviate the neuronal cell death induced by $\mathrm{MeHg}$.

\section{Discussion}

$\mathrm{MeHg}$, a toxic heavy metal, may cause neurological disorders. In the current study, $\mathrm{MeHg}$ induced a concentration-dependent increase of cell death in $\mathrm{SH}$ SY5Y cells and rat cerebral cortical neurons. In addition to cell death, certain metals, such as cadmium and manganese can induce autophagy in neuronal cells ${ }^{32-34}$. Autophagy induction had been found to be activated in $\mathrm{MeHg}$-treated human neural stem cells ${ }^{35}$. In the current study, MeHg increased LC3-II protein expression in a concentration- and time-dependent manner in neuronal cells; furthermore, western blot analysis of LC3-II protein expression under baf A1-treated condition showed that autophagy induction increased at $4 \mathrm{~h}$ and $24 \mathrm{~h}$ after $\mathrm{MeHg}$ exposure. These findings suggest that $\mathrm{MeHg}$ induces neuronal cell death and activates autophagy induction.

There is a close relationship between autophagy and cell death $^{36}$. Through autophagy induction and autophagic degradation of damaged protein and organelle, autophagy can alleviate cell injury. It has been reported that autophagy plays a protective role in metals (such as cadmium and manganese)-induced neurotoxicity ${ }^{7,8}$. In the current cell model, after $\mathrm{MeHg}$ activates autophagy induction to form autophagosome, efficient degradation of autophagy substrates is essential for alleviation of $\mathrm{MeHg}$-induced neurotoxicity. However, we found that $\mathrm{MeHg}$ increased p62 expression that is the index used for detection of lysosomal degradative function ${ }^{37}$, and the autophagic inducers could decrease p62 expression in normal control cells, but this effect was abated in MeHg-treated cells; moreover, $\mathrm{MeHg}$ induced lysosomal membrane permeabilization (LMP) (data not shown). These results indicate that lysosomal degradative function is impaired by $\mathrm{MeHg}$. Under this condition, increased autophagy induction would elicit excessive autophagosome accumulation ${ }^{11-14}$. Consistent with this inference, in the current study, we found that activating or inhibiting the autophagy induction by pharmacological methods increased or decreased autophagosome accumulation in MeHg-exposed neuronal cells. More importantly, activating or inhibiting the autophagy induction had the corresponding effect on cell death. Thus, increased autophagy induction by MeHg promotes autophagosome accumulation and the neuronal cell death. Several studies have reported that autophagosome accumulation is detrimental to cells under stress and pathological situations $^{38,39}$. Accumulation of autophagosomes could result in overproduction of ROS. Moreover, the energy deficit resulting from impaired autophagic degradation also contributes to autophagosome accumulation-induced cytotoxicity $^{40}$. It seems that autophagosome accumulation elicits cell death via different mechanisms, which needs further study.

Autophagy induction is regulated by mTOR-dependent pathway or mTOR-independent pathway ${ }^{15}$. However, the pathway which regulates the MeHg-induced autophagy is still unknown. The mTOR- dependent autophagy is negatively regulated by $\mathrm{mTOR}^{16}$. Our results showing that mTOR substrates were activated in $\mathrm{MeHg}$-treated neuronal cells suggest that the MeHg-induced autophagy is mTOR-independent.

It has been reported that the Vps34 complex is critical for autophagy induction in mTOR-independent autophagy. Beclin1 binds to Vps34 to form Vps34 complex and start autophagy ${ }^{18}$. By combined use of co-immunoprecipitation and immunofluorescence technology, we show for the first time that $\mathrm{MeHg}$ enhances the Beclin1-Vps34 complex formation. Furthermore, chemically synthesized siRNAs against Vps34 inhibited autophagy induction. These results 


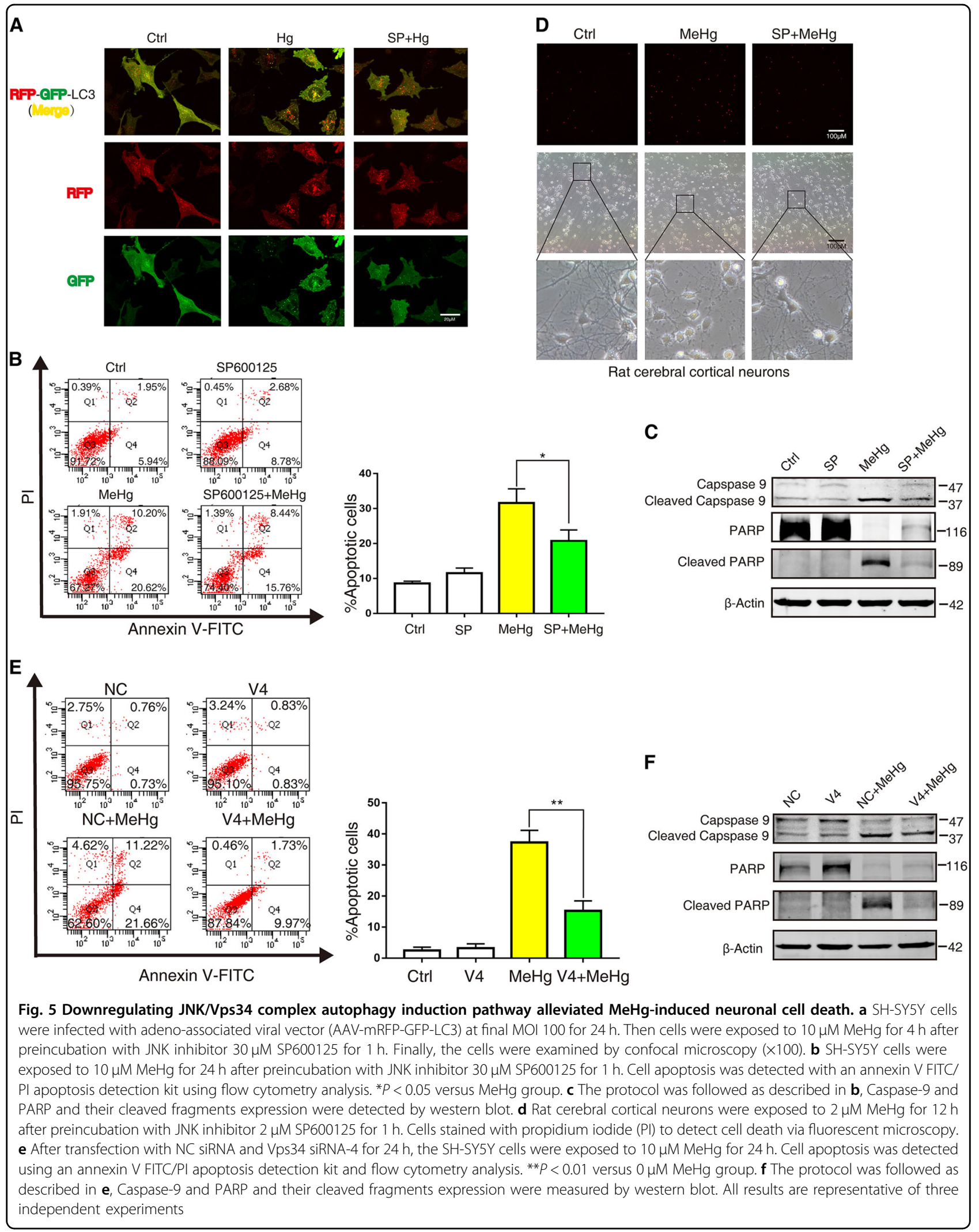




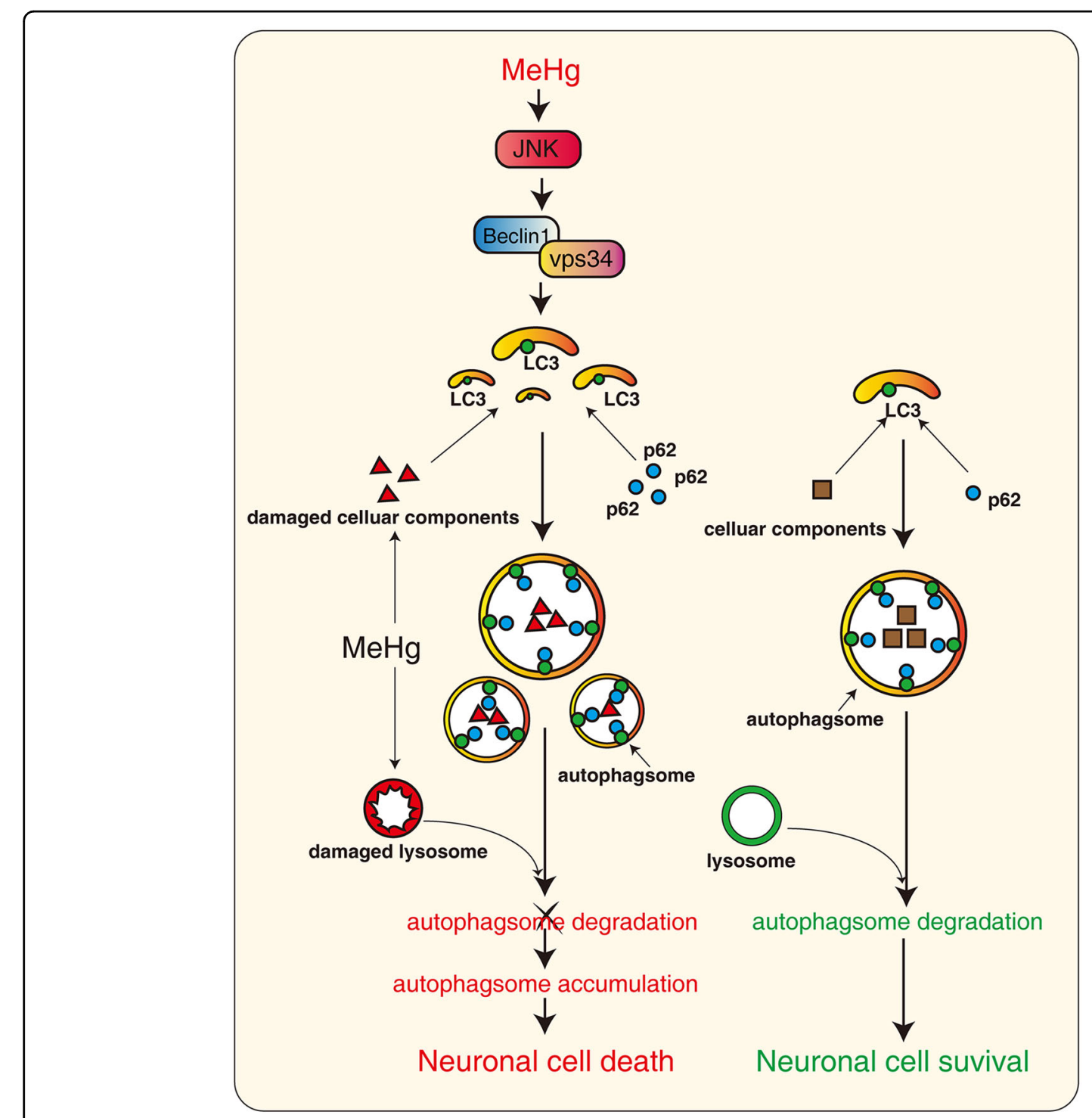

Fig. 6 MeHg-activated autophagy induction through the JNKNps34 complex pathway elicits autophagosome accumulation and neuronal cell death

indicate that $\mathrm{MeHg}$ increases mTOR-independent autophagy induction through enhancing forming the Vps34 complex in neuronal cells.

MAPKs can regulate mTOR-independent autophagy ${ }^{15,30,41}$ and are reported to play an important role in MeHg-induced neurotoxicity ${ }^{42,43}$. We found that MAPKs (JNK, p38, and ERK) were activated by MeHg in neuronal cells. JNK, but not p38 or ERK, promotes the interaction between Beclin1 and Vps34 and autophagy induction in MeHg-treated neuronal cells. It is unclear how JNK regulates the formation of Vps34 complex. Further studies are needed to address this issue. Moreover, Inhibition of JNK or downregulation of Vps34 can decrease autophagosome accumulation and alleviate $\mathrm{MeHg}$-induced neuronal cell death.
Several reports have suggested that calcium dyshomeostasis ${ }^{44,45}$, oxidative stress ${ }^{46,47}$, and disruption of the cytoskeleton $^{48}$ are important mechanisms accounting for MeHg-induced neurotoxicity. How do these mechanistic signals regulate JNK-activated autophagy induction? It has been reported that $\mathrm{Ca}^{2+}$ signal and reactive oxygen species (ROS) may mediate sustained JNK activation through CaMKII ${ }^{49-51}$ and ASK $1^{52,53}$, respectively, and $\mathrm{Ca}^{2}$ + signal, ROS, and phagophore membranes origination can be found at endoplasmic reticulum (ER)-mitochondria contact sites ${ }^{51,54,55}$. These reports raise the possibility that local $\mathrm{Ca}^{2+}$ signal and ROS might regulate JNKactivated autophagy induction and be involved in $\mathrm{MeHg}$ induced neurotoxicity. Moreover, calcium dyshomeostasis and oxidative stress may contribute to impaired 
autophagic degradation. High $\mathrm{Ca}^{2+}$ signaling inhibits the autophagosome-lysosome fusion ${ }^{56,57}$ and oxidative stress leads to lysosomal dysfunction ${ }^{58,59}$. These potential mechanisms need further extensive study.

In summary, we demonstrated that $\mathrm{MeHg}$-induced autophagy, via JNK/Vps34 complex pathway, promotes autophagosome accumulation and neuronal cells death. These findings would help to understand autophagy in the context of MeHg-induced neurotoxicity (Fig.6), and inhibiting autophagy induction pathway might be a novel therapeutic approach for the treatment of MeHg-induced neurotoxicity.

\section{Materials and methods \\ Cell culture and reagents}

Human neuroblastoma cells SH-SY5Y were obtained from the Institute of Biochemistry and Cell Biology (Shanghai, China) and cultured in RPMI 1640 medium supplemented with 10\% foetal bovine serum (both Invitrogen, Carlsbad, CA). Primary neuronal cultures were prepared from one-day-old Sprague-Dawley rat. Firstly, cerebral cortex was dissected and chopped, then incubated with $0.125 \%$ trypsin $/ 10 \%$ EDTA solution at $37^{\circ} \mathrm{C}$ for $10 \mathrm{~min}$. The digestion was stopped with FBS. A sterile fire-polished Pasteur pipette was used to triturate the cells gently for about 20 times and then cells were collected through centrifuging at $800 \mathrm{rpm}$ for $5 \mathrm{~min}$. Cells were suspended in DMEM/F12 Medium containing $10 \%$ FBS and plated at a density of $2.2 \times 10^{5} \mathrm{cells} / \mathrm{cm}^{2}$ in plates pre-coated with poli-L-lysine (Sigma-Aldrich, P-1524). DMEM/F12 Medium was changed to Neurobasal Medium (Gibco, A3582901) supplemented with $2 \%$ of B27 (Gibco, A3582801), 0.5mM L-glutamine, $100 \mu \mathrm{g} / \mathrm{ml}$ penicillin/streptomycin after $4 \mathrm{~h}$ of incubation. The neurobasal medium was refreshed by exchanging half of the volume with fresh media 3 days later. Cells were cultured for 7 days at $37^{\circ} \mathrm{C}$ in a humidified $5 \% \mathrm{CO}_{2} / 95 \%$ air atmosphere. $\mathrm{MeHg}$ treatment was initiated at 7 days.

$\mathrm{MeHg}$ was purchased from Dr.Ehrenstorfer (Germany). Wortmannin, Torin1, SP600125, U0126, SB203580 were purchased from Selleckchem (Houston, TX, USA). LC3 A/B, Beclin1, Vps34, Caspase-9, P-P70s6k Thr389, PP70s6k Ser371, T-P70s6k, P-4EBP1, T-4EBP1, $\beta$-Actin were purchased from Cell Signaling Technologies (Danvers, MA, USA) for western blot. Beclin1, Vps34 were purchased from Bioss (China Beijing) for Immunofluorescence.

\section{Apoptosis assessment}

MeHg-induced apoptosis was measured by flow cytometry using an annexin V-FITC/PI apoptosis detection kit (KeyGEN BioTECH Jiangsu China). Following digestion, the cells were washed twice in ice-cold phosphate- buffered saline (PBS) at a concentration of $5 \times 10^{5}-1 \times$ $10^{6} / \mathrm{ml}$ by centrifugation at $600 \times g$ for $5 \mathrm{~min}$ and resuspended in $500 \mu \mathrm{l}$ binding buffer. Then, $5 \mu \mathrm{l}$ annexin $\mathrm{V}$ FITC and $5 \mu$ propidium iodide (PI) were added and the samples were placed in the dark for $15 \mathrm{~min}$ followed by immediate analysis using a FACSCanto II flow cytometer with BD FACSDiva software v6.1.3 (both Becton Dickinson, San Jose, CA). PI as a nuclear and chromosome counterstain that is not permeant to live cells, and annexin $\mathrm{V}$, which binds to the apoptosis marker phosphatidylserine was added to the samples to distinguish necrotic (annexin $\mathrm{V}-$, PI+), late apoptotic events (annexin $\mathrm{V}+, \mathrm{PI}+$ ) from early apoptotic events (annexin $\mathrm{V}+, \mathrm{PI}-)$. MeHg-induced death of the cerebral cortical neurons was detected using a fluorescent microscope (Nikon ECLIPSE Ti). The cell death rate was calculated as the number of PI+cells/total number of cells.

\section{Knockdown of Vps34}

Four specific siRNAs (small interfering RNAs) against different Vps34 sites were obtained from GenePharma Co. (Shanghai, China) with the following sequences: siRNA-1 sense strand: CACCAAUGAAGCUGAAUAA TT, antisense strand: UUAUUCAGCUUCAUUGGUGT T; siRNA-2 sense strand: GGCUGAAACUACCAGUAAATT, antisense strand: UUUACUGGUAGUUUCAGCCTT; siRNA-3 sense strand: CUGGAUAGAUU GACAUUUATT, antisense strand: UAAAUGUCAAUCUAUCCAGTT; siRNA-4 sense strand: GGCAUUGCUUGGAGAUAAUTT, antisense strand: AUUAUCUCC AAGCAAUGCCTT. Scrambled siRNA was used as a negative control (NC) (NC sense strand: UUCUCCGAACGUGUCACGUTT, antisense strand: ACGUGACACGUUCGGAGAATT). The siRNA was introduced into the cells using Lipofectamine RNAiMAX Transfection Reagent (Invitrogen) according to the manufacturer's instructions.

\section{Western blot analysis}

The proteins were separated by sodium dodecyl sulphate-polyacrylamide gel electrophoresis (SDS PAGE) and transferred onto a PVDF membrane (Millipore Immobilon-FL). The membranes were incubated for $1 \mathrm{~h}$ at room temperature in blocking buffer followed by overnight incubation at $4{ }^{\circ} \mathrm{C}$ in blocking buffer containing the primary antibody. Then, they were washed three times before incubation with the secondary antibody for $1 \mathrm{~h}$ at room temperature. The signal was detected using an Odyssey Infrared Imaging System (LI-COR Biosciences, Lincoln, NE).

\section{Co-immunoprecipitation (Co-IP)}

The cells were cultured in a $100-\mathrm{mm}$ dish. After the designated treatments, they were collected, washed with 
ice-cold PBS, incubated in lysis buffer for $20 \mathrm{~min}$ on ice, and clarified via high-speed $(13,000 \times g)$ centrifugation at $4{ }^{\circ} \mathrm{C}$ for $30 \mathrm{~min}$. The supernatants were incubated overnight at 4 with specific primary antibodies as required followed the addition of $80 \mu \mathrm{l}$ of Protein G Plus/Protein A Agarose Suspension (Merck Millipore, Darmstadt, Germany) and incubation with gentle rotation at $4{ }^{\circ} \mathrm{C}$ for $2 \mathrm{~h}$. The agarose beads were collected and washed five times with lysis buffer and resuspended in $20 \mathrm{ml}$ of $2 \times$ SDS loading buffer. The samples were analysed by western blot.

\section{Immunofluorescence}

For the immunofluorescence studies, $5 \times 10^{5}$ rat cerebral cortical neurons were seeded on a $35-\mathrm{mm}$ confocal plate. After the designated treatments, the cells were washed three times with PBS and fixed in $4 \%$ paraformaldehyde for $15 \mathrm{~min}$ at room temperature. Then, the cells were passed through frozen methanol for $10 \mathrm{~min}$ at $-20{ }^{\circ} \mathrm{C}$ and blocked in 5\% BSA for $30 \mathrm{~min}$. The cells were incubated overnight at $4{ }^{\circ} \mathrm{C}$ with the appropriate primary antibody (1:100-1:200) in 5\% BSA and with the secondary antibodies (Alexa Fluor anti-mouse 594 and anti-rabbit 488) (Thermo Fisher) (1:100) in 5\% BSA for $60 \mathrm{~min}$ at room temperature. An Olympus FluoView ${ }^{\text {ma }}$ FV1000 confocal laser scanning microscope with a $\times 100$ objective was used to record the resultant images.

\section{Adenovirus infection}

The cells were infected with the tandem fluorescenttagged adeno-associated viral vector AAV-mRFP-GFPLC3 (Hanbio Biotechnology, Shanghai, China) at a multiplicity of infection of 500 and experimentally treated as indicated. This tagged AAV was utilized to observe the intensity of autophagy flux based on the different $\mathrm{pH}$ stability of RFP and GFP proteins ${ }^{26}$. The relative fluorescence intensity was detected using an Olympus FluoView $^{\text {ma }}$ FV1000 confocal laser scanning microscope with a $\times 100$ objective. GFP, but not mRFP, degrades in an acidic environment. Thus, yellow spots (a mixture of red and green) indicate autophagosomes, whereas red spots indicate autolysosomes (fused phagosome and lysosome). If autophagy is activated and the autophagic flux is normal, the red signal predominates over the yellow signal. If the autophagic flux is impaired, more yellow than red signal will be observed.

\section{Statistical analysis}

Results were expressed as the mean \pm standard deviation (S.D.). Student's $t$-test was used to determine the statistical significance of the difference in values between two groups. One-way ANOVA was used for statistical analysis of the difference in values among multiple groups. $P<0.05$ was considered significant.

\section{Acknowledgements}

Guangdong Natural Science Foundation (No. 2016A030313534); National Natural Science Foundation of China (No. 81741131)

Conflict of interest

The authors declare that they have no conflict of interest.

\section{Publisher's note}

Springer Nature remains neutral with regard to jurisdictional claims in published maps and institutional affiliations.

Supplementary Information accompanies this paper at (https://doi.org/ 10.1038/s41419-019-1632-z).

Received: 17 January 2019 Revised: 21 April 2019 Accepted: 6 May 2019 Published online: 21 May 2019

\section{References}

1. Karita, K. et al. Recent epidemiological studies on methylmercury, mercury and selenium. Nihon Eiseigaku Zasshi. Jpn. J. Hyg. 71, 236-251 (2016).

2. Fischer, R. G., Rapsomanikis, S., Andreae, M. O. \& Baldi, F. Bioaccumulation of methylmercury and transformation of inorganic mercury by macrofungi. Environ. Sci. Technol. 29, 993-999 (1995).

3. Aschner, M. \& Aschner, J. L. Mercury neurotoxicity: mechanisms of blood-brain barrier transport. Neurosci. Biobehav. Rev. 14, 169-176 (1990).

4. Rice, D. C., Schoeny, R. \& Mahaffey, K. Methods and rationale for derivation of a reference dose for methylmercury by the US EPA. Risk Anal. 23, 107-115 (2003).

5. Kroemer, G., Mariño, G. \& Levine, B. Autophagy and the integrated stress response. Mol. Cell 40, 280-293 (2010).

6. Orrenius, S., Kaminskyy, V. O. \& Zhivotovsky, B. Autophagy in toxicology: cause or consequence? Annu. Rev. Pharmacol. Toxicol. 53, 275-297 (2013).

7. Wang, Q. W. et al. Cadmium-induced autophagy promotes survival of rat cerebral cortical neurons by activating class III phosphoinositide 3-kinase/ beclin-1/B-cell lymphoma 2 signaling pathways. Mol. Med. Rep. 12, 2912-2918 (2015).

8. Zhang, J. et al. The role of autophagy dysregulation in manganese-induced dopaminergic neurodegeneration. Neurotox. Res. 24, 478-490 (2013).

9. Denton, D. \& Kumar, S. Autophagy-dependent cell death. Cell Death Differ. 26, 605-616 (2019).

10. Bialik, S., Dasari, S. K. \& Kimchi, A. Autophagy-dependent cell death-where, how and why a cell eats itself to death. J. Cell Sci. 131, jcs215152 (2018).

11. Wan, J. et al. Inhibition of miR-155 reduces impaired autophagy and improves prognosis in an experimental pancreatitis mouse model. Cell death \& disease 10, 303 (2019).

12. Wang, $X$. et al. The roles of oxidative stress and Beclin-1 in the autophagosome clearance impairment triggered by cardiac arrest. Free Radic. Biol. Med. https://doi.org/10.1016/j.freeradbiomed.2018.12.039 (2019).

13. Bordi, M. et al. Autophagy flux in CA1 neurons of Alzheimer hippocampus: Increased induction overburdens failing lysosomes to propel neuritic dystrophy. Autophagy 12, 2467-2483 (2016).

14. Elrick, M. J., Yu, T., Chung, C. \& Lieberman, A. P. Impaired proteolysis underlies autophagic dysfunction in Niemann-Pick type C disease. Hum. Mol. Genet. 21, 4876-4887 (2012).

15. Sarkar, S. Regulation of autophagy by mTOR-dependent and mTORindependent pathways: autophagy dysfunction in neurodegenerative diseases and therapeutic application of autophagy enhancers. Biochem Soc Trans. 41, 1103-1130 (2013).

16. Jung, C. H., Ro, S.-H., Cao, J., Otto, N. M. \& Kim, D.-H. mTOR regulation of autophagy. FEBS Lett. 584, 1287-1295 (2010).

17. Guan, H. et al. 2, 5-Hexanedione induces autophagic death of VSC4. 1 cells via a PI3KJAkt/mTOR pathway. Mol. Biosyst. 13, 1993-2005 (2017).

18. Backer, J. M. The intricate regulation and complex functions of the Class III phosphoinositide 3-kinase Vps34. Biochem. J. 473, 2251-2271 (2016).

19. Arsikin, K. et al. Autophagy-dependent and-independent involvement of AMPactivated protein kinase in 6-hydroxydopamine toxicity to SH-SY5Y neuroblastoma cells. Biochim. Biophys. Acta 1822, 1826-11836 (2012). 
20. Lu, Z. et al. Colistin-induced autophagy and apoptosis involves the JNK-BCl2Bax signaling pathway and JNK-p53-ROS positive feedback loop in PC-12 cells. Chem. Biol. Interact. 277, 62-73 (2017).

21. Zhao, W. et al. iTRAQ proteomics analysis reveals that PI3K is highly associated with bupivacaine-induced neurotoxicity pathways. Proteomics 16, 564-575 (2016).

22. Su, P. et al. Genistein alleviates lead-induced neurotoxicity in vitro and in vivo: Involvement of multiple signaling pathways. Neurotoxicology 53, 153-164 (2016).

23. Wang, H., Engstrom, A. K. \& Xia, Z. Cadmium impairs the survival and proliferation of cultured adult subventricular neural stem cells through activation of the JNK and p38 MAP kinases. Toxicology 380, 30-37 (2017).

24. Mizushima, N. \& Yoshimori, T. How to interpret LC3 immunoblotting. Autophagy 3, 542-545 (2007).

25. Eskelinen, E.-L. \& Saftig, P. Autophagy: a lysosomal degradation pathway with a central role in health and disease. Biochim. Biophys. Acta 1793, 664-673 (2009)

26. Duan, N.-N., Liu, X.-J. \& Wu, J. Palmitic acid elicits hepatic stellate cell activation through inflammasomes and hedgehog signaling. Life Sci. 176, 42-53 (2017).

27. Zeng, X.-J. et al. Impaired autophagic flux is associated with the severity of trauma and the role of A 2A R in brain cells after traumatic brain injury. Cell Death Dis. 9, 252 (2018).

28. Duan, P. et al. 4-Nonylphenol induces autophagy and attenuates mTORp70S6K/4EBP1 signaling by modulating AMPK activation in Sertoli cells. Toxicol. Lett. 267, 21-31 (2017).

29. Henson, S. M. et al. p38 signaling inhibits mTORC1-independent autophagy in senescent human CD8+T cells. J. Clin. Investig. 124, 4004-4016 (2014).

30. Ugland, H., Naderi, S., Brech, A., Collas, P. \& Blomhoff, H. K. cAMP induces autophagy via a novel pathway involving ERK, cyclin $\mathrm{E}$ and Beclin 1. Autophagy 7, 1199-1211 (2011).

31. Kim, M.-O., Lee, H.-S., Chin, Y.-W., Moon, D.-O. \& Ahn, J.-S. Gartanin induces autophagy through JNK activation which extenuates caspase-dependent apoptosis. Oncol. Rep. 34, 139-146 (2015).

32. Gorojod, R. et al. The autophagic-lysosomal pathway determines the fate of glial cells under manganese-induced oxidative stress conditions. Free Radic. Biol. Med. 87, 237-251 (2015).

33. Wang, Q. et al. Induction of cytoprotective autophagy in PC-12 cells by cadmium. Biochem. Biophys. Res. Commun. 438, 186-192 (2013).

34. Wang, T. et al. The ER stress regulator Bip mediates cadmium-induced autophagy and neuronal senescence. Sci. Rep. 6, 38091 (2016).

35. Chang, S.-H. et al. Methylmercury induces caspase-dependent apoptosis and autophagy in human neural stem cells. J. Toxicol. Sci. 38, 823-831 (2013).

36. Green, D. R. \& Levine, B. To be or not to be? How selective autophagy and cell death govern cell fate. Cell 157, 65-75 (2014).

37. Bjørkøy, G. et al. Monitoring autophagic degradation of p62/SQSTM1. Methods Enzymol. 452, 181-197 (2009).

38. Sarkar, C. et al. Impaired autophagy flux is associated with neuronal cell death after traumatic brain injury. Autophagy 10, 2208-2222 (2014).

39. Saleem, S. \& Biswas, S. C. Tribbles pseudokinase 3 induces both apoptosis and autophagy in amyloid- $\beta$-induced neuronal death. J. Biol. Chem. 292, 2571-2585 (2017).
40. Button, R. W., Roberts, S. L., Willis, T. L., Hanemann, C. O. \& Luo, S. Accumulation of autophagosomes confers cytotoxicity. J. Biol. Chem. M117, 782276 (2017).

41. Wang, Q. \& Ren, J. mTOR-Independent autophagy inducer trehalose rescues against insulin resistance-induced myocardial contractile anomalies: role of p38 MAPK and Foxo1. Pharmacol. Res. 111, 357-373 (2016).

42. Fujimura, M. \& Usuki, F. Site-specific neural hyperactivity via the activation of MAPK and PKA/CREB pathways triggers neuronal degeneration in methylmercury-intoxicated mice. Toxicol. Lett. 271, 66-73 (2017).

43. Guida, N. et al. p38/Sp1/Sp4/HDAC4/BDNF axis is a novel molecular pathway of the neurotoxic effect of the methylmercury. Front. Neurosci. 11 8 (2017).

44. Sarafian, T. A. Methyl mercury increases intracellular Ca2+and inositol phosphate levels in cultured cerebellar granule neurons. J. Neurochem. 61, 648-657 (1993).

45. Roos D, Seeger R, Puntel R. et al. Role of calcium and mitochondria in MeHgmediated cytotoxicity. J Biomed Biotechnol (2012).

46. Farina $M$, Aschner $M$. Methylmercury-induced neurotoxicity: focus on prooxidative events and related consequences. Adv Neurobiol 18, 267-286 (Springer, Germany, 2017).

47. Antunes dos Santos, A. et al. Oxidative stress in methylmercury-induced cell toxicity. Toxics 6, 47 (2018).

48. Pierozan, P. et al. Neurotoxicity of methylmercury in isolated astrocytes and neurons: the cytoskeleton as a main target. Mol. Neurobiol. 54, 5752-5767 (2017).

49. Brnjic, S., Olofsson, M. H., Havelka, A. M. \& Linder, S. Chemical biology suggests a role for calcium signaling in mediating sustained JNK activation during apoptosis. Mol. Biosyst. 6, 767-774 (2010).

50. Liu, A.-J. et al. Evodiamine, a plant alkaloid, induces calcium/JNK-mediated autophagy and calcium/mitochondria-mediated apoptosis in human glioblastoma cells. Chem. Biol. Interact. 205, 20-28 (2013).

51. Bootman, M. D., Chehab, T., Bultynck, G., Parys, J. B. \& Rietdorf, K. The regulation of autophagy by calcium signals: do we have a consensus? Cell Calcium 70 32-46 (2018).

52. Kadowaki, $H$. et al. Amyloid $\beta$ induces neuronal cell death through ROS mediated ASK1 activation. Cell Death Differ. 12, 19 (2005).

53. Matsuzawa, A. \& Ichijo, H. Redox control of cell fate by MAP kinase: physiological roles of ASK1-MAP kinase pathway in stress signaling. Biochim. Biophys. Acta 1780, 1325-1336 (2008).

54. Marchi, S. et al. Mitochondrial and endoplasmic reticulum calcium homeostasis and cell death. Cell Calcium 69, 62-72 (2018).

55. Bagur, R. et al. Arsenic targets local ROS and calcium homeostasis at the mitochondria-ER interface. Biophys. J. 114, 659a (2018).

56. Corona, A.K. \& Jackson, W.T. Finding the middle ground for autophagic fusion requirements. Trends Cell Biol. 28, 869-881 (2018).

57. Liu, F. et al. Cadmium disrupts autophagic flux by inhibiting cytosolic Ca2 +-dependent autophagosome-lysosome fusion in primary rat proximal tubular cells. Toxicology 383, 13-23 (2017).

58. Scherz-Shouval, R. \& Elazar, Z. Regulation of autophagy by ROS: physiology and pathology. Trends Biochem. Sci. 36, 30-38 (2011).

59. Tai, H. et al. Autophagy impairment with lysosomal and mitochondrial dysfunction is an important characteristic of oxidative stress-induced senescence Autophagy 13, 99-113 (2017). 